\title{
X-Ray Variability in LINERs
}

\author{
Hernández-García L. ${ }^{1}$, González-Martín O. ${ }^{2}$, Masegosa $\mathrm{J}^{1}{ }^{1}$ and \\ Márquez, $\mathbf{I}^{1}$ \\ ${ }^{1}$ Instituto de Astrofísica de Andalucía \\ Glorieta de la Astronomía, s/n 18008, Granada, Spain \\ ${ }^{2}$ Instituto de Astrofísica de Canarias, \\ C/ Vía Láctea s/n 38205 La Laguna, Tenerife, Spain \\ email: lorena@iaa.es
}

\begin{abstract}
Active galactic nuclei (AGN) are powered by energetic phenomena which cannot be attributed to stars. LINERs appears to be the low power end of the AGN sequence with the lowest Eddington ratios but hosting the most massive black holes (typically $10^{9} \mathrm{M}_{\odot}$ ). Whereas variability is common for high Eddington ratio emitting sources, in the low Eddington regime data are not so clear. Recent investigations at UV (Maoz et al. 2005) and X-ray frequencies (Younes et al. 2011, González-Martín et al. 2011) point out to the long term variable nature for some particular LINERs.

In this work we add more evidence about the X-ray variability in LINERs and investigate its nature. The data set comprises X-ray archival spectroscopy from observations taken from Chandra and XMM-Newton, selecting LINERs with several observations at different epochs. Up to now we analysed two LINER 1.9 objects, NGC 1052 and NGC 4278, and checked that variability is due to different mechanisms based on the results of spectral fittings.
\end{abstract}

Keywords. Galaxies, AGN, LINERs, variability, X-ray

\section{Introduction}

LINERs were classified in the optical using diagnostic diagrams (Heckman 1980, Ho et al. 1997). These diagrams represents emission line intensity ratios, being the spectra of LINERs dominated by low ionisation lines. Although there is controversy, it is thought that LINERs may be the low power end of the AGN sequence, having the lowest Eddington ratios and the most massive black holes (BHs). At X-rays we can asses the AGN nature of a source if it is detected at hard X-rays. In our previous work, 82 LINERs with Chandra and/or XMM-Newton data for single period observations were analysed, concluding that more than $80 \%$ of the objects hosted AGN (González-Martín et al. (2006, 2009)).

Now we deal with variability, since it is one of the main properties of AGNs. McHardy et al. (2006) found that variability time scale increases for bigger mass of the $\mathrm{BH}$ and/or lower bolometric luminosities. Because LINERs have high BH masses and low bolometric luminosities, this puts them into a variability time scale of months to years. Furthermore, González-Martín \& Vaughan (2012) found that LINERs do not vary in time scales lower than tens of days. The first evidence of variability was reported by Maoz et al. (2005), where all but three objects in the sample of 17 LINERs 1 and 2 were variable in the UV. More recently, Younes et al. (2011) studied type 1 LINERs in X-rays and concluded that variability is a common property in these objects at long-term periods. 
Table 1. Observational details.

\begin{tabular}{cccc|cccr}
\hline \multicolumn{3}{c}{ NGC 1052 } & \multicolumn{5}{c}{ NGC 4278 } \\
\hline Satellite & OBSID & Date & $\begin{array}{c}\text { Exptime } \\
(\mathrm{ksec})\end{array}$ & Satellite & OBSID & Date & $\begin{array}{r}\text { Exptime } \\
(\mathrm{ksec})\end{array}$ \\
\hline & & & & & & & \\
& & & & & & \\
$X M M$-Newton & 093630101 & $2001-08-15$ & 16.3 & Chandra & 4741 & $2005-02-03$ & 37.9 \\
$X M M$-Newton & 306230101 & $2006-01-12$ & 54.9 & Chandra & 7077 & $2006-03-16$ & 111.7 \\
$X M M$-Newton & 553300301 & $2009-01-14$ & 52.3 & Chandra & 7078 & $2006-07-25$ & 52.1 \\
$X M M$-Newton & 553300401 & $2009-08-12$ & 59.0 & Chandra & 7079 & $2006-10-24$ & 106.4 \\
& & & & Chandra & 7081 & $2007-02-20$ & 112.1 \\
& & & & Chandra & 7080 & $2007-04-20$ & 56.5 \\
\hline
\end{tabular}

\section{Sample and Data Reduction}

We have identified 16 objects from the sample of González-Martín et al. (2009) which show hints of variability. Here we present the results for two LINERs 1.9: NGC 1052 and NGC 4278. We used the HEASARC $†$ archive to search different observations with Chandra (ACIS-S) and XMM-Newton (EPIC pn), with public observations until January 2012. The observational details are summarised in Table 1. Only observations with enough exposure time to have good spectral quality are taking into account.

The reduction and analysis were made in a systematic, uniform way using CXC Chandra Interactive Analysis of Observations (CIAO $\ddagger$ ), version 4.3, for Chandra data, and the Science Analysis Software (SAS $\uparrow$ ), version 11.0.0, for XMM-Newton data. Both reductions were made analogously, eliminating background flares, extracting the nuclear source and the background from circular regions and grouping spectra. The response matrix (RMF) and ancillary reference files (ARF) were generated for each source region.

\section{Analysis and Results}

We fit all the spectra from the same object with the same model of XSPEC (v. 12.7.0) to identify which parameters are changing and to get clues on the nature of the observed variations. For this purpose we used five different models. Note that we used two absorbers when two components were present in the model (following the same prescription given by Gonzalez-Martin et al. 2009): (a) MEKAL: A pure thermal model; (b) PL: A single power law; (c) 2PLs: Two power laws; (d) MEPL: A composite of a thermal plus a single power law; (e) ME2PL: A composite of a thermal plus two power laws.

We let different parameters vary in the model: column densities, $\mathrm{N}_{\mathrm{H}}$, slope of the power law, $\Gamma$, normalisations of each component and the temperature of the thermal model, $\mathrm{kT}$.

For NGC 1052, we fit a model containing a thermal plus two power laws components. After varying the parameters in the model, the best fit let the $\mathrm{N}_{\mathrm{H} 2}$ and the normalisation $\left(\mathrm{Norm}_{2}\right)$, associated to the hard energies, vary (Fig. 1, left).

This can be better seen if we plot the values of $\mathrm{N}_{\mathrm{H} 2}$ and the $\mathrm{Norm}_{2}$ of the power law (Fig. 1, right) or the luminosities calculated for this model (Fig. 3, left) for each observation. As expected, there is an anti-correlation between the $\mathrm{N}_{\mathrm{H} 2}$ variations and luminosities, because the higher the absorption, the lower the luminosities. Because $X M M$ Newton has an optical monitor, we can compare these luminosities in X-rays to those in the UV. It is also seen the same behaviour on luminosities (Fig. 3, centre). There are

$\dagger$ http://heasarc.gsfc.nasa.gov/

$\ddagger$ http://cxc.harvard.edu/ciao4.4/

I http://xmm.esa.int/sas/ 
only three observations with the optical monitor, coinciding with the last three dates in $\mathrm{X}$-rays. Both hard $\mathrm{X}$ rays and UV luminosities show variations amounting to $\sim 25 \%$.
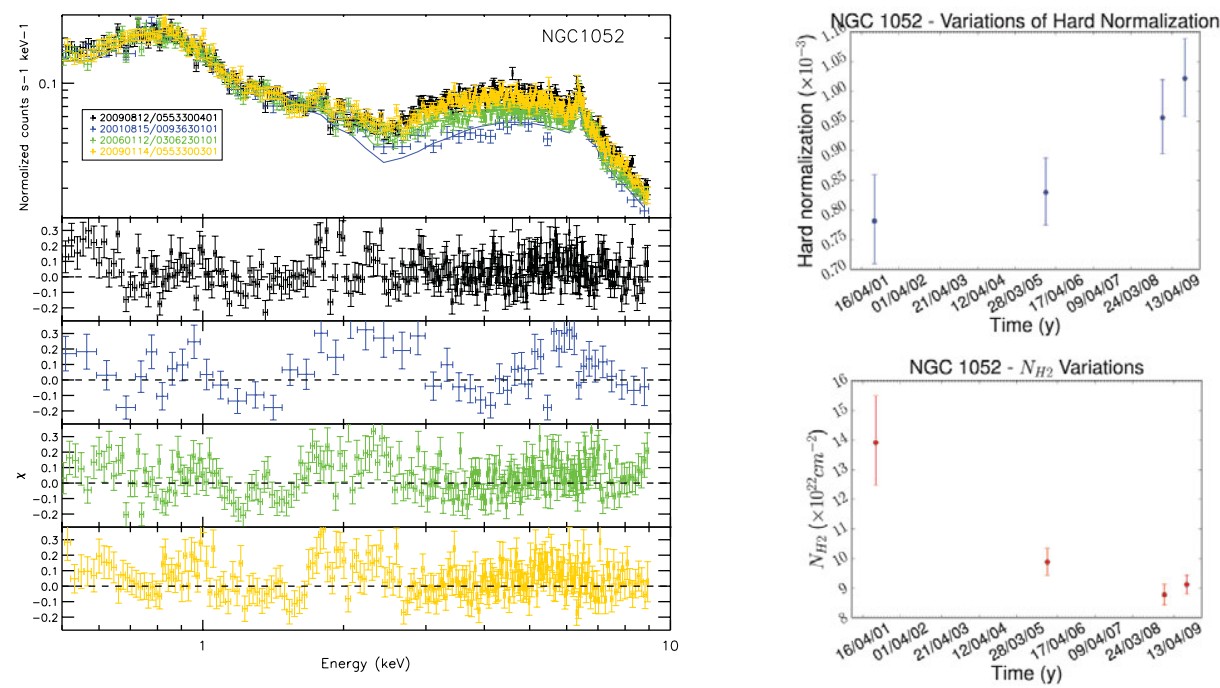

Figure 1. Best fit for NGC 1052 (left), where the variability is due to variations of the $\mathrm{N}_{\mathrm{H} 2}$ and the $\mathrm{Norm}_{2}$ of the PL, associated to high energies (right).

For NGC 4278 we fit a model containing a thermal component plus a single power law (Fig. 2, left). The best fit implies variations of the $\Gamma$ and the Norm of the power law (Fig. 2, right).
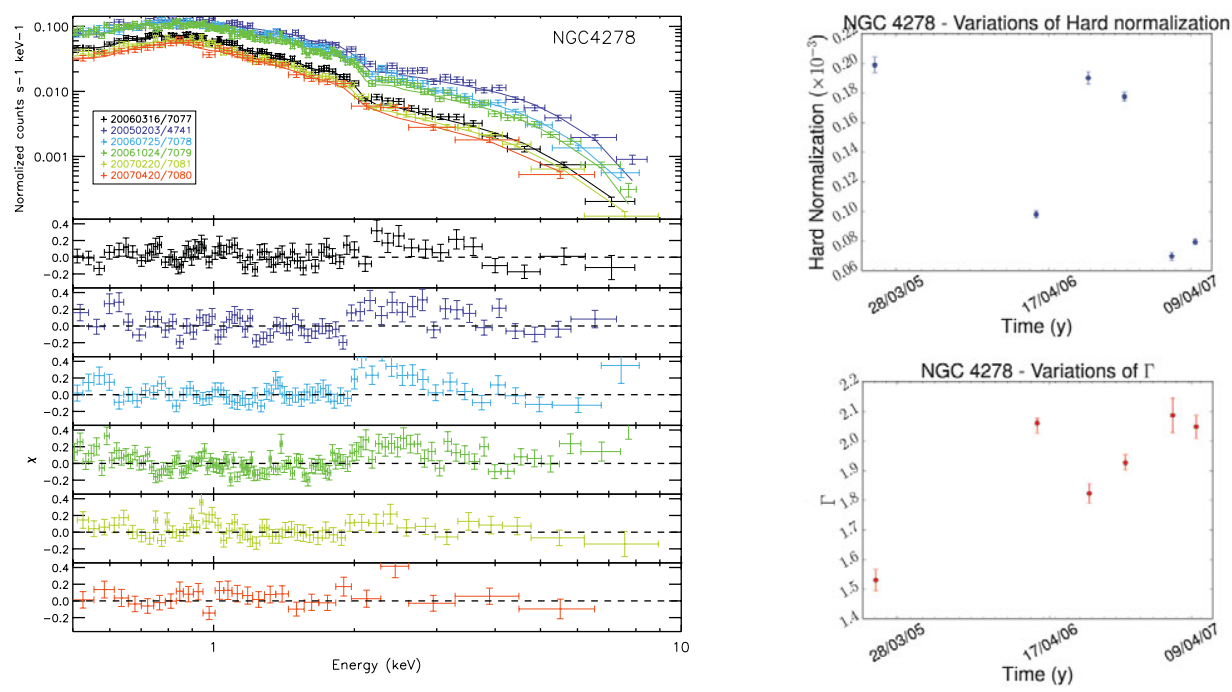

Figure 2. Best fit for NGC 4278 (left), where the variability is due to variations of the $\Gamma$ and the Norm of the power law (right).

Here there is an anti-correlation between the variations of the $\Gamma$ of the PL and the luminosities (Fig. 3, right). In this case we cannot compare the luminosities in the UV at the same dates since only Chandra data are available. However Cardullo et al. (2008), using data from HST, found a UV luminosity increase of a factor of 1.6. 

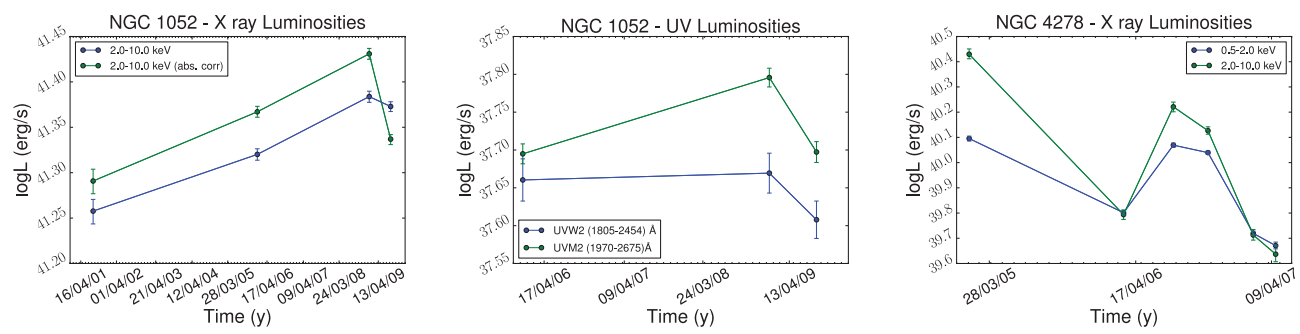

Figure 3. Luminosities calculated for: left) hard X-rays (2-10 keV) for NGC 1052, including absorption corrected luminosity; center) UV from the optical monitor (XMM-OM) with different filters for NGC 1052 and right) Soft (0.5-2 keV) and hard X-rays for NGC 4278.

\section{Discussion and Conclusions}

The scenario for NGC 1052 is consistent with those in Risaliti et al. (2007, 2010, 2011) for different Seyfert galaxies, where the variability is understood as clouds intersecting the line of sight of the observer. This can be conceivable within the framework of the clumpy torus model by Elitzur \& Shlosman (2006) and Nenkova et al. (2008).

The fit by Younes et al. (2010) for NGC 4278 also left vary the $\Gamma$ and the Norm of the power law. The difference with our model is that they used only one column density instead of two, but we can see the same behaviour in both results. About the anticorrelation between the slope and the luminosities, they suggest that this can be due to an Advection Dominated Accretion Flow (ADAF), where the accretion is inefficient. In this case the variability may be attributed to an intrinsic variation on the central engine.

The presence of different mechanisms for LINERs have to be confirmed with a larger sample of LINERs, which is under study. We plan to apply the same kind of analysis to the remaining fourteen LINERs from the sample of González-Martín et al. (2009) which show hints of variability. Studying these objects will allow to know more about the nature of such nuclei.

\section{References}

Cardullo, A., Corsini, E. M., Beifiori, A., Pizzella, A., \& Buson, L. M. 2008, ASPC, 396, 53

Elitzur, M. \& Shlosman, I. 2006, ApJ, 648, L101

González-Martín, O., Masegosa, J., Márquez, I., Guerrero, M., \& Dultzin-Hacyan, D. 2006, $A \& A, 460,45$

González-Martín, O., Masegosa, J., Márquez, I., Guainazzi, M., \& Jiménez-Bailón, E. 2009, $A \mathscr{E} A, 506,1170$

González-Martín, O., Papadakis, I., Braito, V., Masegosa, J., Márquez, I., Mateos, S., AcostaPulido, J. A., Martínez, M. A., Ebrero, J., Esquej,P., O’Brien, P., Tueller, J., Warwick, R. S., \& Watson, M. G. 2011, $A \mathscr{E} A$, 527, A142

González-Martín, O. \& Vaughan, S. 2012, A\& A, 544, A80

Heckman, T. M. 1980, A\&A A, 80, 152

Ho, L. C., Filippenko, A. V., Sargent, W. L. W., \& Peng, C. Y. 1997, ApJS, 112, 391

Maoz, D., Nagar, N. M., Falcke, H., \& Wilson, A. S. 2005, ApJ, 625, 699

McHardy, I. M., Koerding, E., Knigge, C., Uttley, P., \& Fender, R. P. 2006, Nature, 444, 730

Nenkova, M., Sirocky, M. M., Ivezić, Ž., \& Elitzur, M. 2008, ApJ, 685, 147

Risaliti, G., Elvis, M., Fabbiano, G., Baldi, A., Zezas, A., \& Salvati, M. 2007, ApJ, 659, L111

Risaliti, G., Elvis, M., Bianchi, S., \& Matt, G. 2010, MNRAS, 406, L20

Risaliti, G., Nardini, E., Salvati, M., Elvis, M., Fabbiano, G., Maiolino, R., Pietrini, P., \& Torricelli-Ciamponi, G. 2011, MNRAS, 406, L20

Younes, G., Porquet, D., Sabra, B., Grosso, N., Reeves, J. N., \& Allen, M. 2010, A\& A, 517, A33

Younes, G., Porquet, D., Sabra, B., \& Reeves, J. N. 2011, A\&3A, 530, A149 